\title{
Reliability and validity of a modified 8-item Morisky Medication Adherence Scale in patients with chronic pain
}

\author{
Yanfeng Zhang ${ }^{1 \#}$, Rongrong Wang ${ }^{1 \#}$, Qiming Chen ${ }^{1}$, Sizhe Dong ${ }^{1,2}$, Xuejiao Guo ${ }^{1}$, Zhiying Feng ${ }^{1}$, \\ Yuefeng Rao ${ }^{1}$
}

${ }^{1}$ The First Affiliated Hospital, Zhejiang University School of Medicine, Hangzhou, China; ${ }^{2}$ Department of Pharmacy, The First People's Hospital of Nanning, Nanning, China

Contributions: (I) Conception and design: R Wang, Y Rao; (II) Administrative support: None; (III) Provision of study materials or patients: Q Chen, X Guo; (IV) Collection and assembly of data: Y Zhang, Z Feng; (V) Data analysis and interpretation: S Dong; (VI) Manuscript writing: All authors; (VII) Final approval of manuscript: All authors.

"These authors contributed equally to this work.

Correspondence to: Zhiying Feng; Yuefeng Rao. The First Affiliated Hospital, Zhejiang University School of Medicine, Hangzhou 310000 , China. Email: fzy1972@zju.edu.cn; raoyf@zju.edu.cn.

Background The 8-item Morisky Medication Adherence Scale (MMAS-8) is a simple, economic and easy tool to evaluate the medication compliance of chronic disease. The reliability and validity of the MMAS8 in patients with chronic pain were unclear. Therefore, we aimed to validate the MMAS- 8 for detecting nonadherent patients with chronic pain.

Methods: A modified MMAS- 8 was used to assess the medication compliance of patients with chronic pain who were treated at our hospital from July 2018 to October 2018. Cronbach's $\alpha$ was used to evaluate the internal consistency, and a factor analysis was used to examine the construct validity. Convergent validity was assessed by comparing the MMAS-8 and a medication adherence visual analog score (MA-VAS) through Pearson's correlation coefficient.

Results: A total of 113 patients were evaluated. The (t-test) results revealed that there was a significant difference in average scores between the low-score group (who scored less than 5 points) and the high-score group (who scored 8 points or above), indicating that the scale displayed a good degree of discrimination. Except for Items 4 and 5, all the other items exhibited a good correlation with the total score (correlation coefficient $>0.5 ; \mathrm{P}<0.05)$. The Cronbach's $\alpha$ coefficient was 0.625 , indicating that the scale's internal consistency was relatively satisfactory. Two common factors, which explained $62.978 \%$ of the total variance, were extracted by factor analysis to examine the construct validity of the MMAS- 8 , and the load of the 6 items was greater than 0.4. The Pearson correlation coefficient was $0.845(\mathrm{P}<0.001)$; thus, convergent validity was high.

Conclusions: The modified MMAS-8 exhibited acceptable reliability and validity in evaluating medication compliance in patients with chronic pain; thus, it can be applied to detect nonadherent patients with chronic pain.

Keywords: Chronic pain; medication compliance; Morisky Medication Adherence Scale (MMAS); reliability; validity

Submitted Jun 21, 2021. Accepted for publication Aug 10, 2021.

doi: 10.21037/apm-21-1878

View this article at: https://dx.doi.org/10.21037/apm-21-1878 


\section{Introduction}

Pain has been listed as 1 of the 5 major vital signs, including blood pressure, body temperature, breathing, and pulse. At the 2002 World Congress on Pain, the International Association for the Study of Pain expressed the view that chronic pain should be listed as a disease (1). Concerning the disease itself, long-term pain stimulation can lead to the pathological remodeling of the central nervous system and the subsequent prolongation and recalcitrance of the disease. Further, the chronic process of pain also affects patients' work and life (2). Therefore, chronic pain has been recognized as a significant public health problem across the globe.

The management of chronic pain involves multiple combinations of therapeutic methods (3). Effective analgesic pharmacotherapy is critical for pain treatment, with more than $60 \%$ of chronic pain patients receiving analgesic drugs $(3,4)$. Poor medication adherence remains the central problem in managing chronic diseases and is commonly observed in chronic pain patients (4). Evaluation of the medication adherence status in clinical practice is the first step before improving the condition in chronic pain patients (5).

Since the statement by the WHO in 2001, it has been widely accepted that no "gold standard" exists for evaluating therapeutic compliance (6). Direct measurements are recognized as the most accurate; however, the usual invasive procedures limit their use. As an indirect measure, if the questionnaire assesses specific medical recommendations, it may help better predict therapeutic compliance (6). At present, commonly used clinical compliance questionnaires include the Morisky Medication Adherence Scale (MMAS), which Morisky published in 1986 (7) to measure the medication compliance of patients with hypertension, the Medication Adherence Rating Scale (MARS), which was proposed by Thompson et al. in 2000 (8), and the Antidepressant Compliance Questionnaire (ADCQ), which was developed by Demyttenaere et al. (9). The 8 -item MMAS (MMAS-8) scale was initially developed for the hypertensive population (10), and it has better psychometric properties than the original MMAS. Because it is simple, economical, and effective, it is widely used to evaluate medication compliance in patients with chronic diseases (11-14). MMAS-8 was also used in older adults with noncancer chronic pain in a previous study (4). To date, however, there is still a lack of studies that validated the MMAS-8 in evaluating medication compliance in patients with chronic pain. In this study, the reliability and validity of a modified version of the MMAS- 8 were evaluated in outpatients diagnosed with chronic pain. We present the following article in accordance with the SURGE reporting checklist (available at https://dx.doi.org/10.21037/apm-211878).

\section{Methods}

\section{Study subjects}

From July 2018 to October 2018, patients with chronic pain who sought treatment at the outpatient pain clinic of our hospital (the First Affiliated Hospital, Zhejiang University School of Medicine) were recruited to participate in this questionnaire-based study. To be eligible to participate in the study, patients had to meet the following inclusion criteria: (I) be an adult aged over 18 years old who had been diagnosed with chronic pain; (II) be taking painkillers, including nonsteroidal anti-inflammatory drugs, opioid analgesics, and drugs for treating neuropathic pain; and (III) have been taking the prescribed medication for 8 weeks or more. Patients were excluded from the study if they met any of the following exclusion criteria: (I) had communication or cognitive impairment; and/or (II) were unwilling to complete the questionnaires. No incentives were provided to included patients.

\section{Study methods}

A field questionnaire was administered to the subjects who met the recruitment criteria. The questionnaire content included demographic data, a pain visual analog scale, a medication adherence visual analog score (MA-VAS), and the 8-item modified MMAS-8 (see summary in Figure 1 and Table 1). This study was approved by the Ethics Committee of the First Affiliated Hospital, Zhejiang University School of Medicine (Reference Number: 2018[758-1]). The data are anonymous, and the requirement for informed consent was therefore waived. The study conformed to the provisions of the Declaration of Helsinki (as revised in 2013).

\section{Main assessment tools}

\section{PAIN-VAS}

Using a numerical rating scale for pain assessment, participants were asked to classify their pain on a scale of 0 to 10 (on which 0 represented "no" pain and 10 represented 


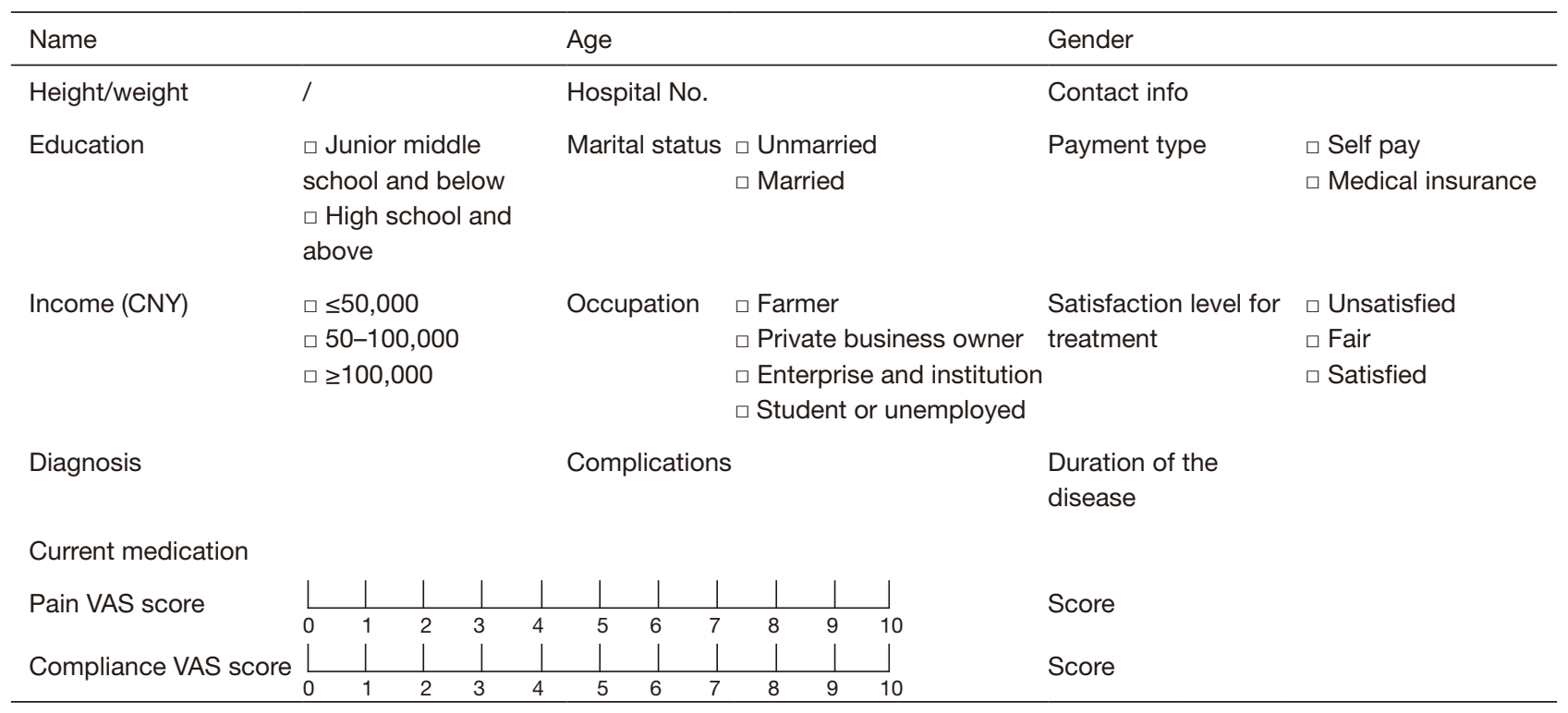

Figure 1 Demographic data of patients with chronic pain. VAS, visual analog score.

"the most severe pain imaginable"). Each participant chose a number that best represented the degree of pain they were experiencing. If a participant was unable to do this, an investigator helped the participant understand the scale and select a number corresponding to the degree of pain they were experiencing. A score of $0-3$ represented mild pain concerning the PAIN-VAS score, a score of 4-6 represented moderate pain, and a score of $7-10$ represented severe pain.

\section{MA-VAS}

Participants were asked to rate their medication compliance on a scale of 10 (on which 0 represented "complete noncompliance" and 10 represented "complete compliance"). Each participant self-evaluated their medication adherence according to the schedule and dosage determined by their doctor and marked the value on a line segment to indicate compliance.

\section{Modified MMAS-8}

First, two professionals, who were specialists in medical English, translated the English version of the MMAS8 (10) into Chinese. In doing so, they replaced the phrase "antihypertensive drugs" with "analgesic drugs". The draft was discussed to reach a consensus. Afterward, a professional, who was a specialist in medical English, translated the Chinese version back into English to evaluate the accuracy of the modified version. The original English scale and the translated Chinese scale were then compared and adjusted. Finally, the scale was tested on 10 individuals. Based on these results, necessary modifications were made to generate the final modified version of the MMAS-8 (see Figure 1 and Table 1). The questionnaire comprised 8 "yes" or "no" items. A score of 1 was allocated for each of Items $1-3$ and 5-8 if a participant answered "no", and a score of 1 was allocated for Item 4 if the participant answered "yes". The total score for the scale was the sum of the scores for each item; the higher the score, the better the medication compliance.

\section{Statistical methods}

The original data were entered in duplicate, and SPSS 22.0 was used to analyze all the respondents. The continuous data are expressed as mean \pm standard deviation or medians (interquartile range) appropriately, and the count data are expressed as ratios. A two-sided $\mathrm{P}<0.05$ indicated a statistically significant difference.

\section{Item analysis}

After dividing the total score for the modified MMAS-8 into quartiles, the low-score group was compared to the high-score group using independent sample $t$-tests, and the presence of significant differences between the two groups was determined. The difference resulting from comparing the two extreme groups was considered the 
Table 1 Modified MMAS-8 for medication compliance of patients with chronic pain

\begin{tabular}{|c|c|c|c|}
\hline No. & Items & Yes & No \\
\hline II & In the past 2 weeks, have you forgotten to use analgesics? & 0 & 1 \\
\hline III & $\begin{array}{l}\text { When you feel that the condition is aggravated or changed, do you adjust the dose without telling your } \\
\text { doctor? }\end{array}$ & 0 & 1 \\
\hline $\mathrm{Vl}$ & $\begin{array}{l}\text { When you feel that your pain is under control, do you stop taking analgesics without consulting your } \\
\text { doctor? }\end{array}$ & 0 & 1 \\
\hline VII & Do you ever find it difficult to adhere to analgesic treatment? & 0 & 1 \\
\hline VIII & Do you find it difficult to remember to take your daily dose of medicine on time? & 0 & 1 \\
\hline
\end{tabular}

MMAS-8, 8-item Morisky Medication Adherence Scale.

critical ratio (CR). If the $\mathrm{CR}$ was not significant for an item, consideration was given as to whether the item should be removed.

\section{Item-total correlations}

The Pearson correlation analysis generated a productmoment correlation coefficient between each item and the total score. Items were adjusted or removed if the correlation coefficients were not significant or were low (correlation coefficient $<0.400$ ).

\section{Reliability analysis}

Cronbach's $\alpha$ was used to evaluate the internal consistency of the modified MMAS-8. The scale was considered unacceptable if Cronbach's $\alpha$ was $<0.60$, acceptable if Cronbach's $\alpha$ was $0.60-0.70(14,15)$, good if Cronbach's $\alpha$ was $0.70-0.80$, and excellent if Cronbach's $\alpha$ was $0.80-0.90$.

\section{Validity analysis}

A factor analysis (a principal component analysis with varimax rotation) was used to examine the construct validity of the modified MMAS-8. The Kaiser-MeyerOlkin (KMO) test, Bartlett's sphericity test, and measures of sampling adequacy (MSA) were used to determine whether the items were suitable for factor analysis. Pearson's correlation coefficient was used to evaluate the convergent validity of the scores for the modified MMAS-8 and the MA-VAS.

\section{Results}

\section{General participant information}

A total of 120 questionnaires were distributed, and 113 valid questionnaires (response rate $=94.2 \%$ ) were retrieved. Among the participants, 47 were male, and 66 were female. Participants' ages ranged from 24 to 87 . The main course of chronic pain was 3-6 months (46\%). There was no difference in the proportions of participants suffering from cancer pain, neuropathic pain, or skeletal muscle pain. The participants used two to three types of painkillers (64.6\%). General information about the participants is set out in Table 2.

\section{Item analysis}

The total MMAS- 8 score was 5 for the lower quartile and 8 for the upper quartile. Thus, the subjects were divided into three groups according to whether they scored $\leq 5$ points, 6-7 points, or 8 points. Levene's test of homogeneity of variance showed that the average scores for the low-score group $(n=27)$ and high-score group $(n=30)$ were statistically significant $(\mathrm{P}<0.001)$. All items, except Item 4 , exhibited statistically significant differences in the inequality of variance $(\mathrm{P}<0.001)$; thus, most of the items exhibited good performance in discriminating between the high- and lowscore groups (see Table 3). The average total score for the participants derived from the modified MMAS- 8 was $6.25 \pm 1.69$. Concerning compliance, $26.0 \%$ of participants reported low compliance, $45.2 \%$ reported intermediate compliance, and $28.8 \%$ reported high compliance. 
Table 2 General participant information ( $\mathrm{n}=113)$

\begin{tabular}{|c|c|c|}
\hline Category & Cases & Percentage (\%) \\
\hline \multicolumn{3}{|l|}{ Gender } \\
\hline Male & 47 & 41.6 \\
\hline Female & 66 & 58.4 \\
\hline \multicolumn{3}{|l|}{ Age (years old) } \\
\hline$\leq 30$ & 2 & 1.8 \\
\hline $31-50$ & 34 & 30.1 \\
\hline $51-70$ & 57 & 50.4 \\
\hline$\geq 71$ & 20 & 17.7 \\
\hline \multicolumn{3}{|l|}{ Education } \\
\hline Junior middle school and below & 89 & 78.8 \\
\hline High school and above & 24 & 21.2 \\
\hline \multicolumn{3}{|l|}{ Concurrent diseases } \\
\hline Yes & 35 & 31 \\
\hline No & 78 & 69 \\
\hline \multicolumn{3}{|l|}{ Course of pain } \\
\hline $3-6$ months & 52 & 46 \\
\hline 6 months -1 year & 13 & 11.5 \\
\hline 1 year and above & 48 & 42.5 \\
\hline \multicolumn{3}{|l|}{ Pain category } \\
\hline Cancer pain & 20 & 17.7 \\
\hline Neuropathic pain & 28 & 24.8 \\
\hline Musculoskeletal pain & 38 & 33.6 \\
\hline Other & 27 & 23.9 \\
\hline \multicolumn{3}{|l|}{$\begin{array}{l}\text { Number of analgesic drugs taken } \\
\text { concurrently }\end{array}$} \\
\hline 1 & 24 & 21.2 \\
\hline 2 & 31 & 27.4 \\
\hline 3 & 42 & 37.2 \\
\hline$\geq 4$ & 16 & 14.2 \\
\hline
\end{tabular}

\section{Item-total correlations}

The correlation between Item 4 and the total score was not statistically significant $(\mathrm{P}=0.156)$. Among the remaining items, the correlation coefficient between Item 5 and the total score was 0.321 , while the correlation coefficients were $>0.5(\mathrm{P}<0.01)$ for all the other items (see Table 3$)$. The results indicated that except for Items 4 and 5 , the items of the modified MMAS-8 exhibited high homogeneity.

\section{Reliability analysis}

The Cronbach's $\alpha$ value for the modified MMAS- 8 was 0.625 ; thus, the reliability of the modified MMAS- 8 was acceptable, but further improvements were required. After deleting Items 4 and 5, Cronbach's $\alpha$ increased to 0.647 and 0.635 , respectively, but the values were not significantly different from the original value. Consequently, it would have been meaningless to delete the two items, and thus the two items were included in the analysis. The results of the analysis are set out in Table 3.

\section{Validity analysis}

\section{Construct validity}

A factor analysis was conducted to evaluate the construct validity of the modified MMAS-8. The closer the KMO value was to 1 , the more common factors there were between the variables. A KMO value for a factor analysis should be at least $>0.5$. As the KMO value of the modified MMAS-8 was 0.539 , a factor analysis could be performed. Bartlett's sphericity test value was $244.059(\mathrm{P}<0.001)$, which indicated that there were common factors among the correlation matrices, and thus the items were suitable for factor analysis. The closer the MSA of each item to 1 , the more suitable it is for factor analysis. If the MSA is $<0.5$, an item is not suitable for factor analysis. The MSA values for Items 5 and 7 were 0.431 and 0.474 , respectively, whereas those for the remaining items were all greater than 0.5. After removing Items 5 and 7, the remaining 6 items were subject to a principal component analysis in which the number of factors was determined by an eigenvalue $\geq 1$. Two common factors were extracted that explained $62.978 \%$ of the total variance. The varimax rotation method was used to analyze the factor load, revealing that the load of 6 items on the principal factor was more than 0.4. Specifically, common factor 1 included Items 1, 2, and 8, while common factor 2 included Items 3, 4, and 6 (see Table 3).

\section{Convergent validity}

The Pearson correlation coefficient between the total score for the modified MMAS- 8 and that for the MA-VAS was $0.845(\mathrm{P}<0.001)$, which indicated high correlations. The results indicated that the modified MMAS- 8 and MA-VAS were equivalent for assessing medication compliance. In addition, the correlation coefficients between Items 1, 2, 3 , 
Table 3 Item analysis, item-total correlations, reliability analysis, and validity analysis of the MMAS-8 scale

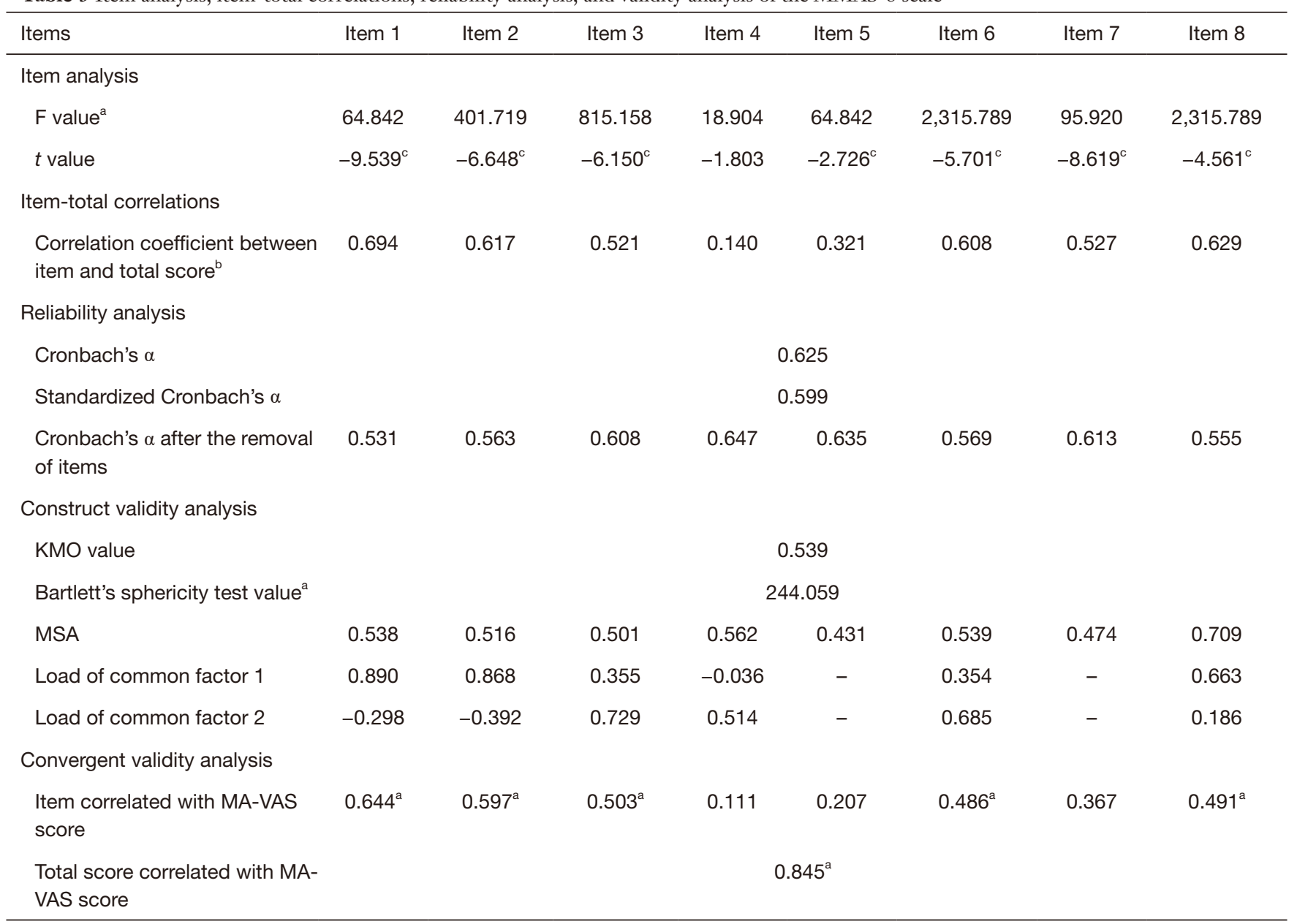

${ }^{\mathrm{a}}, \mathrm{P}<0.001 ;{ }^{\mathrm{b}}, \mathrm{P}<0.01 ;{ }^{\mathrm{c}}, \mathrm{P}<0.05$. MMAS-8, 8-item Morisky Medication Adherence Scale; KMO, Kaiser-Meyer-Olkin; MSA, measures of sampling adequacy; MA-VAS, medication adherence visual analog score.

6 , and 8 in the modified MMAS- 8 with the MA-VAS were all $>0.4(\mathrm{P}<0.001$; see Table 3$)$.

\section{Discussion}

As medication can generate both therapeutic outcomes and toxic side effects during treatment, patients need to strictly follow the advice of medical staff to achieve good outcomes and reduce potential adverse reactions. As such, medication compliance is an important factor affecting medical safety. Chronic pain is characterized by a long duration, slow progression, a large pool of drugs, and complex treatment methods. Such factors often lead to poor medication compliance. This further causes delays in clinical improvement, the extension of treatment duration, and the aggravation of pain.
In 1986, Morisky proposed the MMAS-4 scale to assess patients' medication compliance with hypertension (7) and later expanded it into an 8-item scale. The scale is mainly used for patients with hypertension (10); however, many researchers in China have found that the MMAS scale can be applied to other diseases and displays good reliability and validity in evaluating the medication compliance of patients with a number of diseases, including depression, diabetes, and rheumatoid arthritis (16-18).

To our knowledge, this was the first study to evaluate the reliability and validity of an MMAS-based questionnaire for patients with chronic pain. The results showed that the modified version of the MMAS- 8 displayed overall good performance, reliability, and validity in assessing patients with chronic pain. However, some items did not achieve a good degree of discrimination due to patient 
characteristics or translation issues. For example, Item 4, which sought to examine a patient's medication use when traveling or leaving home for an extended time, exhibited poor homogeneity with the scale's total score. This may be because most of the patients with chronic pain who participated in this study were elderly individuals with physical limitations who were unlikely to leave the house. Concerning reliability, the Cronbach's $\alpha$ in this study was 0.625 , which was lower than expected, and the original MMAS-8 (Cronbach's $\alpha=0.83$ ) (10). Although a Cronbach's $\alpha \geq 0.8$ is recommended, a Cronbach's $\alpha \geq 0.6$ is considered acceptable according to previous studies $(15,16)$. The relatively low Cronbach's $\alpha$ may be due to patients' characteristics, such as the wide age range and the diversity of diseases, and the repetition of some questions. The factor analysis indicated that the modified MMAS- 8 has two dimensions and can reflect two types of nonadherence behaviors in clinical practice, assuming an intentional and an unintentional nonadherence behavior. This structure was different from the original MMAS-8. However, the two extracted common factors can explain $62.978 \%$ of the total variance, and the load of the 6 items was greater than 0.4. This result was consistent with other studies (11-14). Therefore, the modified MMAS- 8 has a structure plausibility. Items 5 and 7 had low and unsatisfactory loads, which may have been due to the cultural background of the Chinese participants and the inability of the questions to capture the actual situations of the patients.

The MMAS questionnaire is convenient and economical; The binary classification of the scale may be overly simplistic and thus fail to elucidate the specific causes of poor medication adherence, especially in assessing the subjective or objective factors that lead to unsatisfactory compliance. According to the information-motivationbehavior (IMB) skills model (19), many factors affect medication behavior, including family supervision, drug use education, and trust in medical staff $(20,21)$. Thus, when evaluating the compliance results of patients in the future, further consideration should be given to patients' motivation and behaviors and other external factors. Some limitations exist. The re-test reliability was not conducted in this study, and we did not monitor the blood concentrations of analgesics, which can be a better criterion-related validity object than the MA-VAS. If such improvements were made, the scale would evaluate better the medication compliance of patients with chronic pain, which would enable clinicians to formulate targeted intervention measures and thus ensure good outcomes.

\section{Acknowledgments}

Funding: This work was supported by the Medical Health Science and Technology Project of Zhejiang Provincial Health Commission [2020KY1036] and the project of Zhejiang Pharmaceutical Association [2019ZYYG02].

\section{Footnote}

Reporting Checklist: The authors have completed the SURGE reporting checklist. Available at https://dx.doi. org/10.21037/apm-21-1878

Data Sharing Statement: Available at https://dx.doi. org/10.21037/apm-21-1878

Conflicts of Interest: All authors have completed the ICMJE uniform disclosure form (available at https://dx.doi. org/10.21037/apm-21-1878). All authors report that this work was supported by the Medical Health Science and Technology Project of Zhejiang Provincial Health Commission [2020KY1036] and the project of Zhejiang Pharmaceutical Association [2019ZYYG02]. The authors have no other conflicts of interest to declare.

Ethical Statement: The authors are accountable for all aspects of the work in ensuring that questions related to the accuracy or integrity of any part of the work are appropriately investigated and resolved. This study was approved by the Ethics Committee of the First Affiliated Hospital, Zhejiang University School of Medicine (Reference Number: 2018[758-1]). The data are anonymous, and the requirement for informed consent was therefore waived. The study conformed to the provisions of the Declaration of Helsinki (as revised in 2013).

Open Access Statement: This is an Open Access article distributed in accordance with the Creative Commons Attribution-NonCommercial-NoDerivs 4.0 International License (CC BY-NC-ND 4.0), which permits the noncommercial replication and distribution of the article with the strict proviso that no changes or edits are made and the original work is properly cited (including links to both the formal publication through the relevant DOI and the license). See: https://creativecommons.org/licenses/by-nc-nd/4.0/.

\section{References}

1. Breivik H. International Association for the Study of Pain: 
update on WHO-IASP activities. J Pain Symptom Manage 2002;24:97-101.

2. Světlík S, Hronová K, Bakhouche H, et al. Pharmacogenetics of chronic pain and its treatment. Mediators Inflamm 2013;2013:864319.

3. Broekmans S, Vanderschueren S. Concerns about medication and medication adherence in patients with chronic pain recruited from general practice. Evid Based Nurs 2012;15:42-3.

4. Timmerman L, Stronks DL, Groeneweg JG, et al. Prevalence and determinants of medication non-adherence in chronic pain patients: a systematic review. Acta Anaesthesiol Scand 2016;60:416-31.

5. Sampaio R, Azevedo LF, Dias CC, et al. Portuguese version of the Medication Adherence Report Scale (MARS-9): Validation in a population of chronic pain patients. J Eval Clin Pract 2019;25:346-52.

6. World Health Organization. Adherence to long-term therapies: evidence for action. Geneva: World Health Organization, 2003.

7. Morisky DE, Green LW, Levine DM. Concurrent and predictive validity of a self-reported measure of medication adherence. Med Care 1986;24:67-74.

8. Thompson K, Kulkarni J, Sergejew AA. Reliability and validity of a new Medication Adherence Rating Scale (MARS) for the psychoses. Schizophr Res 2000;42:241-7.

9. Demyttenaere K, Bruffaerts R, Albert A, et al. Development of an antidepressant compliance questionnaire. Acta Psychiatr Scand 2004;110:201-7.

10. Morisky DE, Ang A, Krousel-Wood M, et al. Predictive validity of a medication adherence measure in an outpatient setting. J Clin Hypertens (Greenwich) 2008;10:348-54.

11. Moharamzad Y, Saadat H, Nakhjavan Shahraki B, et al. Validation of the Persian Version of the 8-Item Morisky Medication Adherence Scale (MMAS-8) in Iranian Hypertensive Patients. Glob J Health Sci 2015;7:173-83.

12. Wang J, Bian R, Mo Y. Validation of the Chinese version of the eight-item Morisky medication adherence scale in

Cite this article as: Zhang Y, Wang R, Chen Q, Dong S, Guo X, Feng Z, Rao Y. Reliability and validity of a modified 8-item Morisky Medication Adherence Scale in patients with chronic pain. Ann Palliat Med 2021;10(8):9088-9095. doi: 10.21037/apm21-1878 patients with type 2 diabetes mellitus. Journal of Clinical Gerontology and Geriatrics 2013;4:119-22.

13. Laghousi D, Rezaie F, Alizadeh M, et al. The eight-item Morisky Medication Adherence Scale: validation of its Persian version in diabetic adults. Caspian J Intern Med 2021;12:77-83.

14. Bowling A. Research methods in health: investigating health and health services. 2nd ed. Buckingham: Open University Press, 2002.

15. Robinson JP, Shaver PR, Wrightsman LS. Measures of personality and social psychological attitudes. San Diego: Academic Press, 1991.

16. Bao LJ, Hu ZH, Xiao JW, et al. Validity and reliability of the Morisky questionnaire in assessing medication compliance in patients with major depressive disorder. J Clin Psychol Med 2014;24:380-2.

17. Wang J, Mo Y, Bian R. Evaluation of reliability and validity of application of the Chinese version of 8-item Morisky Medication Adherence Scale in patients with type 2 diabetes. Chin J Diabetes 2013;21:1101-4.

18. Fan WU, Zhao J, Wang T, et al. Analysis of reliability and validity of the Chinese version Morisky Medication Adherence Scale-8 in assessing medication compliance of the patients with rheumatoid arthritis. China Pharmacy 2018;29:263-8.

19. Liu Z, Li X. Application of Information-MotivationBehavioral Skills Model in behavior intervention. Chinese Journal of Health Education 2016;32:733-5.

20. Timmerman L, Stronks DL, Groeneweg G, et al. The value of medication-specific education on medication adherence and treatment outcome in patients with chronic pain: a randomized clinical trial. Pain Med 2016;17:1829-37.

21. Zhang X, Zheng M, Yuan S. Analysis on patients' compliance and its influential factors. China Pharmacy 2006;17:791-3.

(English Language Editor: J. Huleatt) 